\title{
Original Research Prothesis
}

\section{Longevity of metal-ceramic crowns cemented with self-adhesive resin cement: a prospective clinical study}

\author{
Lucas Pradebon BRONDANI(a) \\ Tatiana PEREIRA-CENCI(a) \\ Vinicius Felipe WANDSHER ${ }^{(\mathbf{b})}$ \\ Gabriel Kalil PEREIRA(b) \\ Luis Felipe VALANDRO(c) \\ César Dalmolin BERGOLI(c) \\ (a) Universidade Federal de Pelotas - UFPel, \\ School of Dentistry, Post-graduate Program \\ in Dentistry, Pelotas, RS, Brazil. \\ (b) Universidade Federal de Pelotas - UFPel, \\ School of Dentistry, Department of Dental \\ Prosthesis, Pelotas, RS, Brazil. \\ (c) Universidade Federal de Santa \\ Maria - UFSM, School of Dentistry, \\ Post-graduate Program in Dentistry, Santa \\ Maria, RS, Brazil.
}

Abstract: Resin cements are often used for single crown cementation due to their physical properties. Self-adhesive resin cements gained widespread due to their simplified technique compared to regular resin cement. However, there is lacking clinical evidence about the long-term behavior of this material. The aim of this prospective clinical trial was to assess the survival rates of metal-ceramic crowns cemented with self-adhesive resin cement up to six years. One hundred and twenty-nine subjects received 152 metal-ceramic crowns. The cementation procedures were standardized and performed by previously trained operators. The crowns were assessed as to primary outcome (debonding) and FDI criteria. Statistical analysis was performed using Kaplan-Meier statistics and descriptive analysis. Three failures occurred (debonding), resulting in a 97.6\% survival rate. FDI criteria assessment resulted in scores 1 and 2 (acceptable clinical evaluation) for all surviving crowns. The use of self-adhesive resin cement is a feasible alternative for metal-ceramic crowns cementation, achieving high and adequate survival rates.

Keywords: Resin Cements; Crowns; Cementation; Survival Rate.

\section{Introduction}

that they have no commercial or associative interest that represents a conflict of interest in connection with the manuscript.

Corresponding Author:

César Dalmolin Bergoli

E-mail: cesar.bergoli@ufpel.edu.br

http://doi.org/10.1590/1807-3107BOR-2017.vol31.0022

Submitted: May 18, 2016

Accepted for publication: Feb 13, 2017

Last revision: Feb 23, 2017
The placement of a metal-ceramic crown is an established restorative option in dentistry for the restoration of vital and/or endodontically treated teeth based on its combination of strength (metal) and esthetics (porcelain) ${ }^{1}$. This type of restoration also functions desirably, with a 5-year survival estimate of $95.6 \%^{2}$. However, to achieve good survival results, a proper cementation technique is needed. An ideal cementing agent should have several characteristics, including biocompatibility, low water sorption and solubility, adhesion, radiopacity, esthetics, easy handling, and low cost; in addition, it must prevent microleakage and resist forces during oral function. ${ }^{3}$ Given that no dental cement currently available fulfills all of the foregoing requirements, the search for an ideal cement material has been the focus of studies., ${ }^{4,5}$

For decades, zinc phosphate cement was used in dentistry as the gold standard for cementation procedures, as it showed good clinical performance even after 10 years ${ }^{6,7}$. However, it also included some negative characteristics, such as high solubility, pulp irritation, and microleakage ${ }^{3}$. More recently, 
the advent of self-adhesive resin cements has provided a material that combines beneficial mechanical properties with an easier cementation technique, as this cement allows adhesion to the tooth structure using a simple protocol and thus eliminates multiple clinical steps, i.e., etching, rinsing, drying, and priming/bonding of dental substrates. Therefore, self-adhesive cements represent an interesting alternative to the broadly used conventional systems. ${ }^{8}$ Although there is good evidence indicating that self-adhesive cement is a good alternative for the cementation of glass fiber posts' ${ }^{9}$, sufficient clinical evidence is lacking with respect to their use for the cementation of metal-ceramic crowns, and, as a result, there is no consensus among clinicians. ${ }^{10}$

The majority of clinical trials on metal-ceramic crowns are retrospective and show good survival rates for restoration, ${ }^{11,12}$ but other factors may influence the survival of the restoration apart from the material itself, such as a subject's risk factors (e.g., risk of caries) and hygiene habits. ${ }^{13}$ Thus, this study aimed to evaluate, by means of a prospective longitudinal study, the survival rate of metal-ceramic single crowns cemented with self-adhesive cement with a follow-up period of up to 6 years.

\section{Methodology}

This study was a multicenter prospective clinical trial wherein crowns cemented with self-adhesive resin cement were evaluated. The study was approved by the local research and ethics committee of each center where the study was conducted (099/2009 for center A; 0170.1.243.000-09 for center B). The oral health of each subject was assessed, and written informed consent was obtained before enrollment in the study.

Inclusion criteria for the subjects were as follows: good oral hygiene, the absence of parafunctional habits, and at least one tooth needing post-retained rehabilitation using a glass fiber post and single metal-ceramic crown. Subjects were excluded if the tooth of interest abutted a fixed partial prosthesis or a removable partial denture. The study inclusion period was from 2009 to 2015, during which 129 patients were included in the study who received a total of 152 metal-ceramic single crowns. No sample size calculation was performed, as all subjects who received the above-mentioned treatment at the dental clinics were assessed and also because the experimental design was a prospective clinical trial.

All subjects were clinically evaluated and had their health condition reestablished, if necessary. After the initial evaluation, all teeth needing rehabilitation received one fiber post (White Post DC; FGM ${ }^{\circledR}$, Joinvile, Brazil), which was cemented using RelyX U100/U200 $\left(3 \mathrm{M}^{\circledR}\right.$ ESPE, St. Paul, USA) resin cement or RelyX ARC $\left(3 \mathrm{M}^{\circledR}\right.$ ESPE) + Scotch Bond Multi Purpose (SBMP; 3M ESPE) adhesive system according to the manufacturer's recommendations.

Before postcementation, the teeth were radiographed to determine the working length and to enable the selection of the glass fiber post from the system. Teeth were then isolated with a rubber dam and the root canals were prepared to two-thirds of their length, keeping at least $3 \mathrm{~mm}$ for apical sealing. The post was tested, the coronary length of the post was determined, and the excess cut off with a diamond bur using a high-speed hand piece under constant cooling. Next, the core was built up using SBMP adhesive system associated with microhybrid resin composite (Filtek ${ }^{\mathrm{TM}}$ $\mathrm{Z} 250^{\mathrm{TM}}$; 3M ${ }^{\circledast}$ ESPE). The teeth were prepared with diamond burs under water-cooling, following the recommended preparation for a metal-ceramic crown.

The impression procedures were performed using a unitary acrylic resin tray with polyether material (Impregum; 3M ${ }^{\circledR} \mathrm{ESPE}$ ) and sent to the laboratory to manufacture a metal-ceramic crown. If necessary, occlusal adjustments were made, using extra-fine diamond burs followed by abrasive and polishing rubbers, and the crowns were cemented with self-adhesive resin cement (RelyX U100/U200), following the manufacturer's recommendation. Finally, a new radiograph was taken to serve as the control (baseline evaluation).

Subjects were annually recalled for clinical and radiographic examinations up to a 6-year follow-up period. The main outcome evaluated was crown debonding. Secondary outcomes such as surface gloss, surface and marginal staining, color match and translucency, and esthetic anatomical form were also evaluated following the FDI esthetic criteria. ${ }^{14}$ The evaluation was made by one evaluator at each center, and all evaluators were calibrated using the "www.e-calib.info" web site. The measure of 
consistency across evaluators, was calculated and reached a value of 0.89 .

Statistical analysis was performed using theSPSS ${ }^{\circledR} 2$ for Mac ${ }^{\circledR}$ software (SPSS ${ }^{\circledast}$ Inc, Chicago, IL). Descriptive analysis was used to describe subjects included in the study and reasons for failures. The longevity of the posts and teeth was assessed using a Kaplan-Meier [analysis] and the $[\log ]-$ rank test $(\alpha=0.05)$.

\section{Results}

\section{Participants' characteristics}

The mean age of the 114 subjects was 47.8 years, and the group of subjects consisted of 95 women (mean age of 47.4 years) and 19 men (average age of 49.2 years). After 6 years, 15 subjects were lost during follow-up due to withdrawal ( $\mathrm{n}=17$ crowns), resulting in a recall rate of $91.4 \%$ for the period; a total of 135 crowns were evaluated and the mean period of monitoring was 3.1 years. Considering tooth types, 60 were anterior teeth and 75 were posterior (52 premolars and 23 molars) as shown in Table 1. Of those teeth, 69 had no remaining walls, 34 had one remaining coronal wall, 23 had two remaining coronal walls, and 8 had three remaining walls.

\section{Failures}

By the final analysis, three crowns had failed, two of which were situated in the anterior region and one of which was situated in the pre-molar region (Figure 1); there was not a statistically significant difference in crown failure relative to tooth location. All failures observed consisted of decementation of the crown. Two of these crowns presented no remaining walls, whereas the third had one remaining wall. Two of the failed crowns were re-cemented (one tooth that had no remaining walls and one tooth with one remaining wall) using the same materials and techniques as described above. The other crown (for a tooth with no remaining walls) needed to be redone. The survival rate of crowns was $97.7 \%$ after up to 6-years of follow-up (Figure 2). Five teeth failed for other reasons and were neither analyzed nor considered as unsuccessful. Of those five, four root fractures and one post fracture occurred, failures that are not related to the primary outcome being tested.
Table 1. Distribution of restoration evaluated according to patient gender, patient age group, and region.

\begin{tabular}{lcccc}
\hline Variable & Anterior & PreMolar & Molar & Total \\
\hline Famale (age group) & 48 & 45 & 18 & 112 \\
$17-40$ & 5 & 9 & 4 & 18 \\
$41-50$ & 23 & 20 & 5 & 48 \\
$51-60$ & 14 & 12 & 3 & 30 \\
$>60$ & 6 & 4 & 7 & 17 \\
Male (age group) & 12 & 7 & 4 & 23 \\
$17-40$ & 3 & 1 & 1 & 5 \\
$41-50$ & 6 & 1 & 2 & 9 \\
$51-60$ & 1 & - & - & 1 \\
$>60$ & 3 & 4 & 1 & 9 \\
Total & 60 & 52 & 23 & 135 \\
\hline & & & &
\end{tabular}

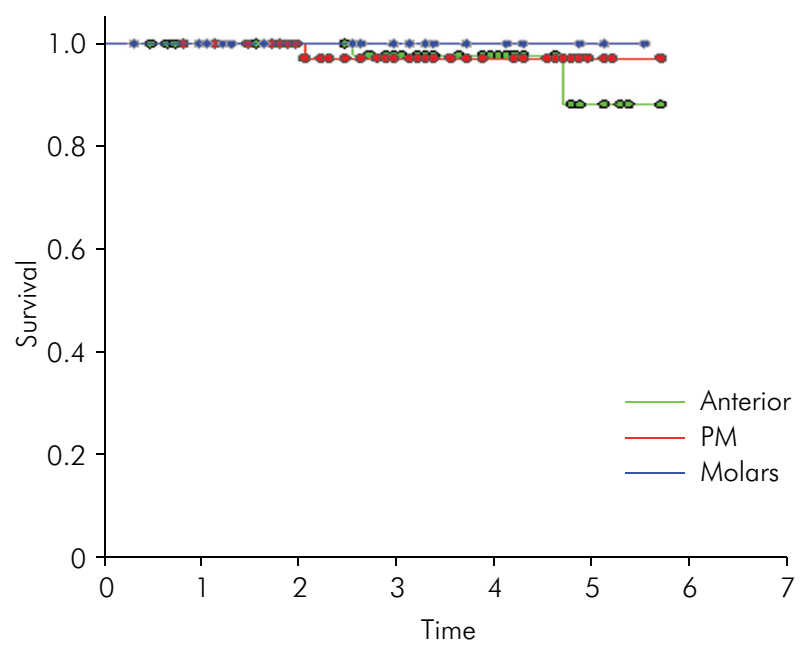

Figure 1. Kaplan Meier survival curve of metal-ceramic single crowns, comparing the region of the crown. Is possible observe that anterior and PM regions presented failures, while molar region did not.

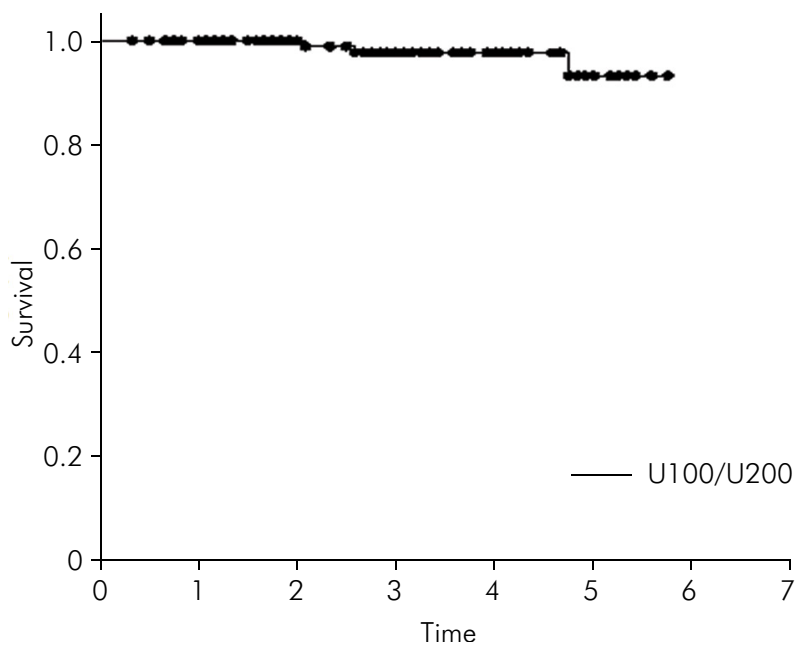

Figure 2. Kaplan Meier survival curve of metal-ceramic single crowns. 


\section{Esthetic parameters}

Concerning the secondary outcomes evaluated, i.e., FDI criteria parameters, which include surface gloss, surface and marginal staining, color match and translucency, and esthetic anatomical form, just one crown received a score of 2 for marginal staining. All the others crowns were evaluated with a score of 1 for all other criteria. Thus, all crowns evaluated were considered acceptable for these parameters after up to 6 years of follow-up.

\section{Discussion}

Self-adhesive resin cements are interesting materials, in particular because of their easy application, which does not require any dentin treatment before use. As a consequence, self-adhesive cements are easier to use in the clinic, as fewer steps for crown cementation are necessary, thus reducing chair-time and costs for the dentist and the patient. These characteristics notwithstanding, clinical scientific evidence is still lacking concerning self-adhesive resin cements, especially with respect to high-quality evidence such as that produced in clinical trials.

The survival rate obtained in this study was $97.7 \%$, which is an excellent rate and is in accordance with a survival rate of $\sim 95.7 \%$ as presented in a recent systematic review by Sailer et al. ${ }^{15}$ However, it was possible to evaluate only the survival of crowns in our study; a regression analysis involving failures and variables such as gender, age, or remaining tooth could not be carried out because of the low failure rates found and the absence of comparison groups. The high survival rate may also be related to the easy handling of the self-adhesive resin cement, which reduces the difficulty of the technique. Additionally, self-adhesive cements have adequate mechanical properties. ${ }^{16}$ In their unpolymerized form, self-adhesive resin cements presents a low $\mathrm{pH}$, which provides a demineralization potential during the polymerization reaction and allows close contact between the resin cement and tooth structure, after polymerization the $\mathrm{pH}$ increase and the mechanical and hydrophobic properties get improved. ${ }^{17}$

Teeth located in the anterior region of the mouth can be submitted to oblique forces, which are less likely to occur for teeth located posteriorly, this difference that suggests there could be more failures of restorations anteriorly located ${ }^{18}$. However, this study did not observe statistically significant differences between regions after an average monitoring period of 3.1 years. Perhaps analysis of a larger sample population and a longer prospective analysis could lead to different results.

According to Pjeturson et al. ${ }^{19}$ the loss of fixed dental prosthesis varies from $0.6 \%$ to $2.7 \%$ after 5 years because of biological complications. In this study, with an up to 6-year follow-up, no biological failures occurred, as debonding caused by caries or loss that was due to periodontal disease did not occur. However, it should be pointed out that the subjects of this study were treated at two university facilities and had annual recalls. This may be a limitation of this study with respect to generalization to a broader population, as subjects treated in dental offices may not be likely to have annual check-ups, which could influence survival rates and result in biological complications.

This study, with a mean time of observation of 3.1 years, which is considered a relatively short follow-up period, is still one of only a few trials reported in the literature that describe the effect of self-adhesive resin cement on metal-ceramic crown restoration. If longer periods of observation are obtained in future studies, perhaps the results will be different, i.e. debonding rates could be higher or biological complications could occur. We note, however, that the results presented here show a success rate of $>95 \%$, which is similar to the findings of Hey et al. ${ }^{20}$, who showed survival rates of metal-ceramic crowns of $>90 \%$ after a 6-year follow-up period in a study using zinc phosphate cement, considered the gold standard cement to be used with metal-ceramic crowns.

In our study, although three failures occurred, only one of them was irreversible, suggesting a better prognosis for subjects with this method in general. The present findings support the study of Piwowarczyk et al. ${ }^{21}$, which indicated that self-adhesive resin cements could be used as a reliable alternative for cementation with similar performance as compared with regular resin cements. It may be said that regular resin cements, because of their clinical sensitivity, still 
need to be proven in clinical trials as a good option for lute metal-ceramic crowns, especially considering that this adhesive system may not be appropriate for lute metal-based crowns.

One of the limitations of this study is the absence of comparison groups, which could have allowed a side-by-side comparison with other resin cements used regularly for crown cementation. We note, however, that our findings showed a much higher success rate than did those of Hey et $\mathrm{al}^{20}{ }^{20}$ regarding the esthetic properties investigated. It is well known that ceramic restorations have better esthetic properties than composite resin restorations. Thus, it may be

\section{References}

1. Lu Y, Chen W, Ke W, Wu S. Nickel-based

(Ni-Cr and Ni-Cr-Be) alloys used in dental restorations may be a potential cause for immune-mediated hypersensitivity. Med Hypotheses. 2009;73(5):716-7. https://doi.org/10.1016/j.mehy.2009.04.041

2. Pjetursson BE, Brägger U, Lang NP, Zwahlen M. Comparison of survival and complication rates of tooth-supported fixed dental prostheses (FDPs) and implant-supported FDPs and single crowns (SCs). Clin Oral Implants Res. 2007;18(Suppl 3):97-113. https://doi.org/10.1111/j.1600-0501.2007.01439.x

3. Rosenstiel SF, Land MF, Crispin BJ. Dental luting agents: a review of the current literature. J Prosthet Dent. 1998;80(3):280-301. https://doi.org/10.1016/S0022-3913(98)70128-3

4. Escribano N, Macorra JC. Microtensile bond strength of self-adhesive luting cements to ceramic. J Adhes Dent. 2006;8(5):337-41.

5. Heintze SD. Crown pull-off test (crown retention test) to evaluate the bonding effectiveness of luting agents. Dent Mater. 2010;26(3):193-206. https://doi.org/10.1016/j.dental.2009.10.004

6. Behr M, Rosentritt M, Wimmer J, Lang R, Kolbeck C, Bürgers R et al. Self-adhesive resin cement versus zinc phosphate luting material: a prospective clinical trial begun 2003. Dent Mater. 2009;25(5):601-4. https://doi.org/10.1016/j.dental.2008.11.003

7. Jokstad A. A split-mouth randomized clinical trial of single crowns retained with resin-modified glass-ionomer and zinc phosphate luting cements. Int J Prosthodont. 2004;17(4):411-6. very difficult to observe changes in the esthetic characteristics as compared with the baseline scores over a short period of time. Still, although these results could be altered by longer follow-up periods, changes in those scores might not be related to the use of self-adhesive resin cement.

\section{Conclusion}

Despite a relatively moderate period of evaluation, self-adhesive resin cement for metal-ceramic crown cementation is a feasible alternative for achieving high survival rates in single crown restoration.

8. Piwowarczyk A, Lauer HC, Sorensen JA. Microleakage of various cementing agents for full cast crowns. Dent Mater. 2005;21(5):445-53. https://doi.org/10.1016/j.dental.2004.07.009

9. Sarkis-Onofre R, Skupien JA, Cenci MS, Moraes RR, Pereira-Cenci $\mathrm{T}$. The role of resin cement on bond strength of glass-fiber posts luted into root canals: a systematic review and meta-analysis of in vitro studies. Oper Dent. 2014;39(1):E31-44. https://doi.org/10.2341/13-070-LIT

10. Ferracane JL, Stansbury JW, Burke FJ. Self-adhesive resin cements: chemistry, properties and clinical considerations. J Oral Rehabil. 2011;38(4):295-314. https://doi.org/10.1111/j.1365-2842.2010.02148.x

11. Reitemeier B, Hänsel K, Kastner C, Weber A, Walter MH. A prospective 10-year study of metal ceramic single crowns and fixed dental prosthesis retainers in private practice settings. J Prosthet Dent. 2013;109(3):149-55. https://doi.org/10.1016/S0022-3913(13)60034-7

12. Rinke S, Kramer K, Bürgers R, Roediger M. A practice-based clinical evaluation of the survival and success of metal-ceramic and zirconia molar crowns: 5-year results. J Oral Rehabil. 2016;43(2):136-44 https://doi.org/10.1111/joor.12348

13. Demarco FF, Corrêa MB, Cenci MS, Moraes RR, Opdam NJ. Longevity of posterior composite restorations: not only a matter of materials. Dent Mater. 2012;28(1):87-101. https://doi.org/10.1016/j.dental.2011.09.003

14. Hickel R, Peschke A, Tyas M, Mjör I, Bayne S, Peters M et al. FDI World Dental Federation: clinical criteria for the evaluation of direct and indirect restorations: update and clinical examples. J Adhes Dent. 2010;12(4):259-72. https://doi.org/10.1007/s00784-010-0432-8 
Longevity of metal-ceramic crowns cemented with self-adhesive resin cement: a prospective clinical study

15. Sailer I, Makarov NA, Thoma DS, Zwahlen M, Pjetursson BE. All-ceramic or metal-ceramic tooth-supported fixed dental prostheses (FDPs)? A systematicreview of the survival and complication rates. Part I: single crowns (SCs). Dent Mater. 2015;31(6):603-23. https://doi.org/10.1016/j.dental.2015.02.011

16. Piwowarczyk A, Lauer HC. Mechanical properties of luting cements after water storage. Oper Dent. 2003;28(5):535-42.

17. Bitter K, Paris S, Pfuertner C, Neumann K, Kielbassa AM. Morphological and bond strength evaluation of different resin cements to root dentin. Eur J Oral Sci. 2009;117(3):326-33. https://doi.org/10.1111/j.1600-0722.2009.00623.x

18. Bru E, Forner L, Llena C, Almenar A. Fibre post behaviour prediction factors. A review of the literature. J Clin Exp Dent. 2013;5(3):e150-3. https://doi.org/10.4317/jced.50619
19. Pjetursson BE, Sailer I, Makarov NA, Zwahlen M, Thoma DS. All-ceramic or metal-ceramic tooth-supported fixed dental prostheses (FDPs)? A systematic review of the survival and complication rates. Part II: multiple-unit FDPs. Dent Mater. 2015;31(6):624-39. https://doi.org/10.1016/j.dental.2015.02.013

20. Hey J, Beuer F, Bensel T, Boeckler AF. Single crowns with CAD/CAM-fabricated copings from titanium: 6-year clinical results. J Prosthet Dent. 2014;112(2):150-4. https://doi.org/10.1016/j.prosdent.2013.09.031

21. Piwowarczyk A, Schick K, Lauer HC. Metal-ceramic crowns cemented with two luting agents: short-term results of a prospective clinical study. Clin Oral Investig. 2011;16(3):917-22. https://doi.org/10.1007/s00784-011-0580-5 